\title{
GMR
}

\section{Effects of kinase insert domain receptor $(K D R)$ gene silencing on the sensitivity of A549 cells to erlotinib}

\author{
W.L. Zhu and Y.H. Liu \\ Department of Respiratory Medicine, Zhengzhou People's Hospital, \\ Zhengzhou, China \\ Corresponding author: Y.H. Liu \\ E-mail: liuyanhong345@126.com \\ Genet. Mol. Res. 14 (4): 15073-15080 (2015) \\ Received April 10, 2015 \\ Accepted July 22, 2015 \\ Published November 24, 2015 \\ DOI http://dx.doi.org/10.4238/2015.November.24.15
}

\begin{abstract}
We investigated the effects of kinase insert domain receptor $(K D R)$ gene silencing on the proliferation of A549 cells and their sensitivity to erlotinib. A KDR small interfering RNA (siRNA) sequence was designed and synthesized; then, it was transfected into A549 cells using Lipofectamine ${ }^{\mathrm{TM}}$ 2000. KDR mRNA and protein expression after KDR gene silencing was detected by reverse transcription polymerase chain reaction and western blotting; the A549 cell cycle was detected by flow cytometry. A 3-(4,5-dimethylthiazol-2-yl)-2,5-diphenyltetrazolium bromide assay and colony formation assay were performed to determine the sensitivity of A549 cells to erlotinib after $K D R$ gene silencing. After $48 \mathrm{~h}$ of $K D R$ gene silencing, there was a significant decrease in $K D R$ gene and protein expression $(\mathrm{P}$ $<0.05$ ). The A549 cell cycle was arrested at the G0/G1 phase, and the number of cells in the $S$ phase decreased; the difference was statistically significant $(P<0.05)$. In the KDR gene silencing group, the sensitivity of A549 cells to erlotinib was significantly enhanced $(P<0.05)$. KDR siRNA
\end{abstract}


can significantly silence the KDR gene in A549 cells, inhibit the proliferation of A549 cells, and enhance their sensitivity to erlotinib.

Key words: Kinase insert domain receptor; siRNA; A549 cells; Lung adenocarcinoma; Erlotinib

\section{INTRODUCTION}

Lung cancer has the highest incidence of all cancers worldwide. Lung squamous cell carcinoma is a histological type of lung cancer that is increasingly common and has a progressively early onset; there may be early lymphatic or blood metastasis, and it is not sensitive to radiotherapy or chemotherapy, which severely reduces the 5-year survival rate (Lortet-Tieulent et al., 2014; Tsuta et al., 2014). Tumor cell proliferation is related to gene regulation, which provides a new and effective way of treating cancer (Santos et al., 2007). Studies have shown that the incidence, invasion, and migration of lung cancer are involved in the activation and deactivation of various genes (lannolo et al., 2011). A study by Cantoni et al. (2012) found that the kinase insert domain receptor $(K D R)$ gene plays an important role in lung adenocarcinoma cell proliferation, which can be used as a new target for cancer therapy. Chemotherapy is critical in the comprehensive treatment of lung cancer, and erlotinib is one of the most common and the most effective chemotherapy drugs for lung cancer (Renouf et al., 2014). In this study, human lung adenocarcinoma A549 cells were used to explore the effect of KDR gene silencing on A549 cell proliferation. Their sensitivity to erlotinib was enhanced after co-treatment with erlotinib, providing a theoretical basis for cotreatment comprising the $K D R$ gene therapy and erlotinib administration. The treatment of lung adenocarcinoma was thereby improved, as reported here.

\section{MATERIAL AND METHODS}

\section{Material}

Human lung squamous carcinoma A549 cells were purchased from the Shanghai Cell Bank ofthe Chinese Academy of Sciences; Roswell Park Memorial Institute (RPMI) 1640 medium, trypsin, and fetal bovine serum were purchased from Gibco (Chicago, IL, USA). Lipofectamine ${ }^{\mathrm{TM}}$ 2000 liposomal transfection reagent and TRIzol were purchased from Invitrogen Corporation, USA, the reverse transcription polymerase chain reaction (RT-PCR) two-step kit was from Beijing SBS Gene Technology Co., Ltd., the complementary DNA (cDNA) synthesis kit was from the Japan TOYOBO Company, the AMV reverse transcription kit was from HangzhouBioer, erlotinib (commodity Mingteluokai) was kindly provided by Roche, small interfering RNA (siRNA) gene fragments were synthesized by the Shanghai GenePharma Company, G418 was purchased from Klontech, USA, KDR mouse anti-human monoclonal antibody was purchased from the Santa Cruz Company, USA, 5\% methyl-thiazolyltetrazolium blue and horseradish peroxidase-conjugated goat anti-mouse secondary antibodies were purchased from the Beijing Zhongshan Company, erlotinib was from AstraZeneca UK Ltd., the flow cytometer was a COULTER XL (Beckman Coulter), the 550 microplate reader was from Biorad, USA, and the biological inverted microscope was an Olympus CKX4. An electrochemiluminescence (ECL) kit was purchased from Thermo, USA, and radio-immunoprecipitation assay (RIPA) protein lysate was purchased from the Jiangsu Beyotime Company. 


\section{siRNA design, synthesis, and transfection}

KDR SiRNA and negative control siRNASCR (scrambled siRNA) sequences have been previously reported (Rinkevich et al., 2012) and were as follows: siRNA: 5'-GCCACCAUGUUCUCUAAUA'I'I'-3' (sense strand) and 5'-UAUUAGAGAACAUGGUGGCAT-3' (antisense strand); negative control siRNASCR: 5'-UUCUCCGAACGUGUCACGU'IT-3' (sense strand) and 5'-ACGUGACACGUUCGGAGAATI'-3' (antisense strand). Chemical synthesis was performed by the Shanghai GenePharma Company. MGC-803 cells were cultured with RPMI 1640 medium containing $10 \%$ fetal bovine serum $\left(37^{\circ} \mathrm{C}, 5 \% \mathrm{CO}_{2}\right.$, saturated humidity). The cells were passaged every 3-4 days and cells in the logarithmic growth phase were used in the experiment. MGC-803 cells were seeded on 24-well plates at a density of $1 \times 10^{5}$ cells/well; when the fusion rate reached $70 \%$, the cells were transfected using the liposome method. The transfection was divided into three groups: cells transfected with pGenesil-1-KDR-siRNA vectors were included in the A549/KDR-siRNA group (the experimental group); cells transfected with randomized controlled vectors were included in the A549/control group (the control group); and untransfected A549 cells were included in the A549 group (the blank group). The RNA interference effect was detected at $48 \mathrm{~h}$ after transfection.

\section{RT-PCR}

The culture medium was discarded, and the cells were washed three times with phosphate-buffered saline (PBS). The TRIzol method was used to extract total RNA. Using the cDNA synthesis kit, cDNA was synthesized by reverse transcription. KDR and the glyceraldehyde 3-phosphate dehydrogenase gene $(G A P D H)$ (internal control) were amplified. The KDR upstream primer was: 5'-CTGGCATGGTCTFCTGTGAAGCA-3'; the downstream primer was: 5'-AATACCAGTGGATGTGATGCGG-3'; and the amplification products were $795 \mathrm{bp}$; the GAPDH upstream primer was: 5'-CGTGGAAGGACTCATGACCA-3'; the downstream primer was: 5'-TCCAGGGGTCTTACTCCTTG-3'; and the amplification product was $509 \mathrm{bp}$. The PCR conditions were as follows: denaturation at $94^{\circ} \mathrm{C}$ for $2 \mathrm{~min} ; 35$ cycles each of denaturation at $94^{\circ} \mathrm{C}$ for $45 \mathrm{~s}$, annealing at $62^{\circ} \mathrm{C}$ for $1 \mathrm{~min}$, and extension at $72^{\circ} \mathrm{C}$ for $1 \mathrm{~min}$; and a final extension of 8 min at $72^{\circ} \mathrm{C}$. Agarose gel electrophoresis (2\%) was performed at a voltage of $100 \mathrm{~V}$ for $45 \mathrm{~min}$. A gel automated imaging and analysis system (France $\mathrm{VI}$ ) was used for absorbance scanning, with the ratio of $K D R$ absorbance/GAPDH absorbance taken as the relative strength of mRNA expression.

\section{Western blotting}

The culture medium was discarded, and the cells were washed three times with PBS; 150 $\mu \mathrm{L}$ precooled RIPA lysis buffer was added; the RIPA Iysis buffer had been previously mixed with 1.5 $\mu \mathrm{L}$ phenylmethylsulfonyl fluoride to obtain a final concentration of $1 \mathrm{mM}$. The steps were performed on ice; then, the solution was centrifuged for $5 \mathrm{~min}$ at $4^{\circ} \mathrm{C}$ and $10,000 \mathrm{X}$ and the supernatant was collected. The protein concentration was determined by the BCA method before denaturation at $99^{\circ} \mathrm{C}$ for $10 \mathrm{~min} ; 50 \mu \mathrm{g}$ total protein was collected for $10 \%$ sodium dodecyl sulfate polyacrylamide gel electrophoresis (SDS-PAGE) electrophoresis, electro-transferred onto a polyvinylidenedifluoride membrane, and sealed in blocking solution for $1 \mathrm{~h}$. The samples were then incubated with KDR primary antibody $(1: 1000)$ at $4^{\circ} \mathrm{C}$ overnight. TBST membrane washing was carried out three times 
for 5 min each time (TBST is a mixture of Tris-buffered saline and Tween 20); 1:5000 horseradish peroxidase-labeled secondary antibody and GAPDH were added and the samples were incubated at $37^{\circ} \mathrm{C}$ for $2 \mathrm{~h}$. After washing with PBS, the samples were investigated using ECL.

\section{Investigation of the cell cycle by flow cytometry}

Cells in each group were collected and washed with cold PBS twice, and cell concentration was adjusted to $1 \times 10^{6} / \mathrm{L}$. Then, the cells were fixed in $70 \%$ cold ethanol $\left(4^{\circ} \mathrm{C}\right)$ for $24 \mathrm{~h}$; after washing, they were incubated with Tris-HCL buffer ( $\mathrm{pH} 7.4$ ) containing $10 \mu \mathrm{g} / \mathrm{mL}$ RNA enzyme for $30 \mathrm{~min}$. Propidium iodide $(50 \mu \mathrm{g} / \mathrm{mL})$ was used for the staining of DNA. DNA content distribution was determined by flow cytometry over $1 \mathrm{~h}$, and the percentage of cells in each cycle was calculated.

\section{3-(4,5-dimethylthiazol-2-yl)-2,5-diphenyltetrazolium bromide (MTT) assay to detect the sensitivity of A549 cells to erlotinib after KDR silencing}

After $48 \mathrm{~h}$ of KDR siRNA transfection, A549 cells were collected in each group and seeded on 96-well plates. Each sample was added to three wells; after adhesion, the cells were cultured with different concentrations of erlotinib $(10,25,50,100$, and $200 \mu \mathrm{M})$. After culturing for $48 \mathrm{~h}, 20$ $\mu \mathrm{L}$ MTT (5 mg/mL) was added to each well; after another 4-h incubation, the supernatant was discarded and the cells were incubated with $200 \mu \mathrm{L} 10 \%$ SDS overnight. After shocking for $15 \mathrm{~min}$, the absorbance value (A value) at $490 \mathrm{~nm}$ was detected using an automatic microplate reader. Inhibition rate $(\%)=(A$ control $-A$ experiment $) /(A$ control $-A$ blank $) \times 100 \%$.

\section{Plate cloning to detect the sensitivity of A549 cells to erlotinib after KDR silencing}

After $48 \mathrm{~h}$ of KDR siRNA transfection, A549 cells were collected in each group and seeded on 6-well plates. Each sample was added to three wells; after adhesion, the cells were cultured with different concentrations of erlotinib (10, 25, 50,100, and $200 \mu \mathrm{M})$. After culturing for 7days, the medium was discarded and the cells were washed twice with PBS. They were then fixed with $95 \%$ ethanol for $10 \mathrm{~min}$, stained with Giemsa dye for $10 \mathrm{~min}$, washed, and dried. The Quantity One software was used to calculate the number of clones. Inhibition rate of colony formation $(\%)=$ 1 - (number of clones in the experimental group / number of clones in the control group) x $100 \%$.

\section{Statistical analysis}

The SPSS14.0 statistical software was used for analysis in this study. Data are reported as means \pm standard deviation. Continuous variables between two groups were compared using the $t$-test; continuous variables among groups were compared using analysis of variance and covariance correction. Two-sided test level $\alpha=0.05$.

\section{RESULTS}

\section{KDR siRNA inhibiting KDR mRNA expression}

The RT-PCR of KDR mRNA expression showed that, compared with the control and blank groups, the bands in the experimental group were significantly narrower, and there were statistically 
significant differences between the experimental group and the control group, and between the experimental group and the blank group (all $\mathrm{P}<0.05$ ), as shown in Figure 1.
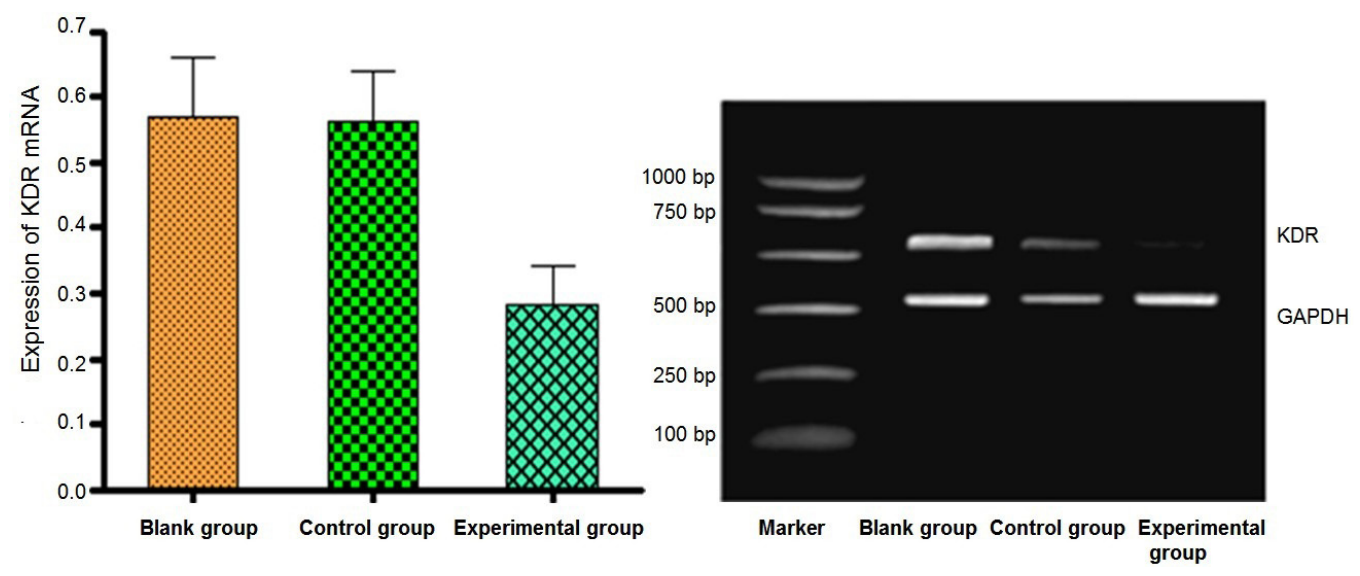

Figure 1. Detection of KDR mRNA expression in A549 cells by RT-PCR.

\section{siRNA inhibiting KDR protein expression}

The western blotting of KDR protein expression showed that, compared with the control and blank groups, bands in the experimental group were significantly narrower, and there were statistically significant differences in the gray values (all $\mathrm{P}<0.05$ ), as shown in Figure 2 .

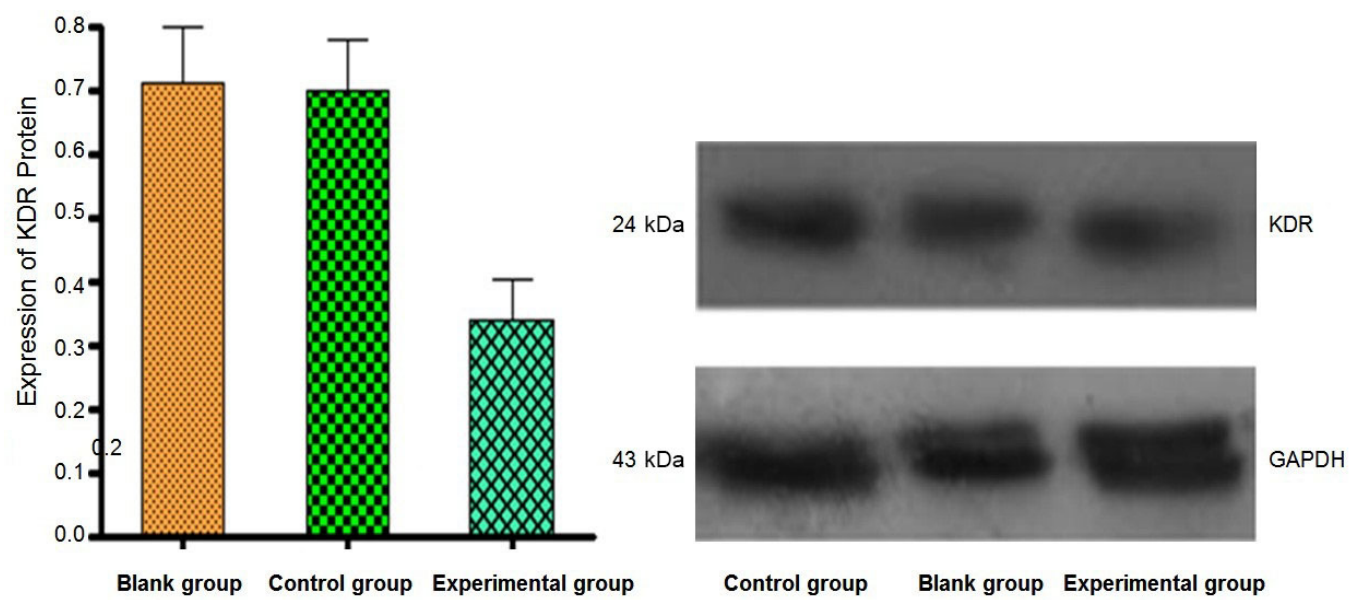

Figure 2. Detection of KDR protein expression in A549 cells by western blot.

\section{Flow cytometry for cell cycle detection}

The A549 cell cycle was investigated by flow cytometry; the results showed that, compared with the control and blank groups, the number of cells in the G0/G1 phase increased slightly and those in the $S$ phase decreased slightly in the experimental group. There were statistically significant differences 
between the experimental and control groups, and between the experimental and blank groups (all $\mathrm{P}$ $<0.05)$. No significant changes were found in the $\mathrm{M}$ phase and the differences were not statistically significant $(P>0.05)$, indicating a significant S-phase arrest in the A549 cells (Table 1 and Figure 3).

Table 1. Effect of small interfering RNA (siRNA)-inhibited kinase insert domain receptor (KDR) expression on the A549 cell cycle (means $\pm S D, N=3$ ).

\begin{tabular}{lllr}
\hline Groups & G0/G1 & S & G2/M \\
\hline Blank & $58.3 \pm 3.7$ & $33.7 \pm 3.3$ & $7.8 \pm 1.7$ \\
Control & $58.2 \pm 3.9$ & $33.8 \pm 4.2$ & $8.6 \pm 1.4$ \\
Experiment & $70.3 \pm 5.6^{*}$ & $20.9 \pm 3.6^{*}$ & $9.3 \pm 2.1$ \\
\hline
\end{tabular}

Compared with the blank and control groups, ${ }^{*} \mathrm{P}<0.05$.

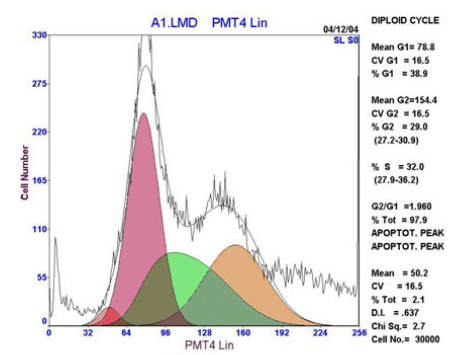

Blank group

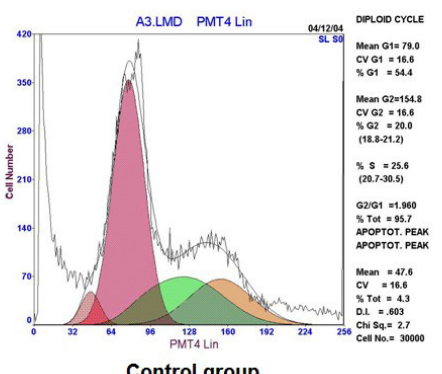

Control group

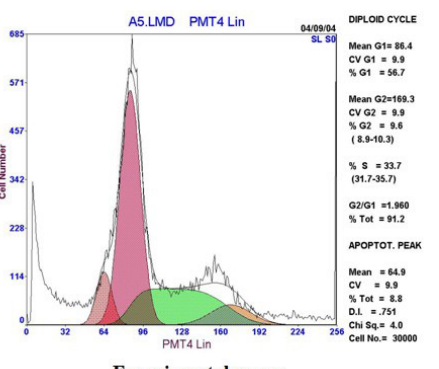

Experimental group

Figure 3. Flow cytometry was used to detect the cell cycle of A549 cells.

\section{Increased sensitivity of A549 cells to erlotinib after KDR interference}

The MTT assay showed that, after pGenesil-1-siRNA-KDR vector transfection, the sensitivity of the A549 cells to different concentrations of erlotinib significantly improved. As drug concentration increased, the inhibition rate of erlotinib for the A549 cells also increased in a dosedependent manner; compared with the control and blank groups, sensitivity of A549 cells to different concentrations of erlotinib significantly increased (all $P<0.05$, Table 2).

Table 2. Inhibition rate of erlotinib on A549 cells detected using the MTT assay aftertransfection ( $\%$, means $\pm S D, N=3$ ).

\begin{tabular}{llrr}
\hline Erlotinib concentration $(\mu \mathrm{M})$ & \multicolumn{2}{c}{ Inhibition rate $(\%)$} \\
\cline { 2 - 4 } & Blank group & Control group & Experimental group \\
\hline 10 & $16.34 \pm 1.57^{*}$ & $16.23 \pm 1.45^{*}$ & $26.45 \pm 1.35$ \\
25 & $22.67 \pm 1.34^{*}$ & $23.23 \pm 1.63^{*}$ & $37.24 \pm 1.35$ \\
50 & $30.85 \pm 2.23^{*}$ & $31.52 \pm 1.36^{*}$ & $50.45 \pm 3.02$ \\
100 & $40.45 \pm 3.23^{*}$ & $41.65 \pm 2.28^{*}$ & $78.52 \pm 2.36$ \\
200 & $50.45 \pm 3.80^{*}$ & $50.42 \pm 3.24^{*}$ & $91.42 \pm 4.68$ \\
\hline
\end{tabular}

MTT = 3-(4,5-dimethylthiazol-2-yl)-2,5-diphenyltetrazolium bromide; ${ }^{*} \mathrm{P}<0.05$ vs the experimental group.

\section{Effect of siRNA KDR transfection on A549 cell colony formation}

The plate clone assay showed that, after pGenesil-1-siRNA-KDR vector transfection, the sensitivity of A549 cells to different concentrations of erlotinib improved; the number of clones significantly reduced and the inhibition rate of colony formation significantly increased $(P<0.05)$. 
With increase in drug concentration, the inhibition rate of cell colony formation also increased in a dose-dependent manner; the inhibition rate of colony formation in the experimental group was significantly higher than those in the control and blank groups (all $\mathrm{P}<0.05$, Table 3 ).

\begin{tabular}{|c|c|c|c|}
\hline \multirow[t]{2}{*}{ Erlotinib concentration $(\mu \mathrm{M})$} & \multicolumn{3}{|c|}{ Clone formation inhibition rate (\%) } \\
\hline & Blank group & Control group & Experimental group \\
\hline 10 & $26.12 \pm 1.23^{*}$ & $26.57 \pm 1.63^{*}$ & $34.45 \pm 1.63$ \\
\hline 25 & $36.42 \pm 1.32^{*}$ & $36.85 \pm 1.25^{*}$ & $46.72 \pm 1.61$ \\
\hline 50 & $46.52 \pm 2.32^{*}$ & $46.42 \pm 2.52^{*}$ & $57.73 \pm 2.64$ \\
\hline 100 & $56.42 \pm 2.01^{*}$ & $56.35 \pm 2.65^{*}$ & $67.52 \pm 2.34$ \\
\hline 200 & $67.32 \pm 2.54^{*}$ & $68.42 \pm 2.52^{*}$ & $79.64 \pm 2.53$ \\
\hline
\end{tabular}

${ }^{*} \mathrm{P}<0.05$ vs the experimental group.

\section{DISCUSSION}

The incidence of lung cancer is increasing annually, and because it is a systemic disease, there may be early lymph or blood metastasis. Moreover, the age of onset is gradually decreasing, seriously threatening human health (Tsuta et al., 2014). Currently, the treatment of lung cancer mainly involves surgery, chemotherapy, radiotherapy, hormonal therapy, biological molecular targeted therapy, or other comprehensive treatment programs, but local recurrence and distant metastasis are still huge problems that plague clinicians. Lung squamous cell carcinoma is a histological type of lung cancer. With the development of functional genomics and completion of the human genome project, cancer gene therapy has become a hot topic in recent years, and attracts a great deal of attention from the medical profession in the field of comprehensive tumor treatment (Rinkevich et al., 2012). The proliferation of lung adenocarcinoma cells is related to the abnormalities of function and structure in various genes. Therefore, identification of the key genes involved in the incidence of lung squamous cell carcinoma is important for the prevention, diagnosis, and treatment of the disease (Gałecki et al., 2013).

In recent years, the development of RNA interference technology has ensured that it has become a focus of interest in cancer gene therapy. Transcribed silencing genes mediated by double-stranded RNA molecules can efficiently and specifically inhibit the expression of various genes associated with cancer incidence and drug resistance, and they are widely used in cancer and genetic research, opening up a new path for the effective treatment of tumors (Curtin et al., 2012). KDR is widely distributed in vascular endothelial cells and plays an important role in their growth and differentiation; it can increase vascular permeability and promote angiogenesis and vascular endothelial cell proliferation, and has become a focus for research in recent years (Adham and Coomber, 2009; Cantoni et al., 2012).Typically, the renewal of vascular endothelial cells is slow, while the proliferation of endothelial cells in tumor tissues is active, and KDR is highly expressed to mediate the generation of vascular endothelial growth factor in tumor angiogenesis (Kim et al., 2006; Holmes and Zachary, 2008). Chemotherapy is critical in the comprehensive treatment of lung cancer, and erlotinib is one of the most common and the most effective chemotherapy drugs for lung cancer (Vickers et al., 2012; Renouf et al., 2014). In this study, KDR siRNA was synthesized and transfected into A549 cells using Lipofectamine ${ }^{\text {TM }}$ 2000. The expression of KDR mRNA and protein after KDR gene silencing was detected by RT-PCR and western blotting; the A549 cell cycle was investigated by flow cytometry. The results showed that after $48 \mathrm{~h}$ of $K D R$ gene silencing, there was 
a significant decrease in $K D R$ gene and protein expression in the A549 cells $(P<0.05)$. The A549 cell cycle was arrested in the G0/G1 phase, and the number of cells in the $S$ phase was reduced; the difference was statistically significant $(P<0.05)$. The MTT assay and colony formation assay were performed to investigate the sensitivity of A549 cells to erlotinib after KDR gene silencing. In the KDR gene silencing group, the sensitivity of A549 cells to erlotinib was significantly enhanced $(P<0.05)$. Therefore, KDR gene silencing in A549 cells can specifically interfere with KDR gene expression, effectively inhibiting the proliferation of human lung adenocarcinoma cells and significantly enhancing chemosensitivity to erlotinib; KDR siRNA sequences provide a new theoretical basis for the treatment of human lung adenocarcinoma (Foster et al., 2009). The combination of $K D R$ gene silencing and erlotinib can effectively induce spontaneous apoptosis in human lung adenocarcinoma A549 cells to some extent, and increases their sensitivity to erlotinib.

In summary, KDR siRNA can significantly silence $K D R$ gene and protein expression in A549 cells, inhibit the proliferation of A549 cells, and affect the growth rate of A549 cells; in combination with erlotinib, there is a synergistic effect, which can enhance the chemosensitivity of A549 cells to erlotinib. Therefore, this study provides a new theoretical basis for the clinical application of a combination of $K D R$ gene silencing and erlotinib in lung cancer treatment.

\section{Conflicts of interest}

The authors declare no conflict of interest.

\section{REFERENCES}

Adham SA and Coomber BL (2009). Glucose is a key regulator of VEGFR2/KDR in human epithelial ovarian carcinoma cells. Biochem. Biophys. Res. Commun. 390: 130-135.

Cantoni S, Cavallini C, Bianchi F, Bonavita F, et al. (2012). Rosuvastatin elicits KDR-dependent vasculogenic response of human placental stem cells through PI3K/AKT pathway. Pharmacol. Res. 65: 275-284.

Curtin ML, Heyman HR, Frey RR, Marcotte PA, et al. (2012). Pyrazolediaminopyrimidines as dual inhibitors of KDR and Aurora B kinases. Bioorg. Med. Chem. Lett. 22: 4750-4755.

Foster KA, Regan HK, Danziger AP, Detwiler T, et al. (2009). Attenuation of edema and infarct volume following focal cerebral ischemia by early but not delayed administration of a novel small molecule KDR kinase inhibitor. Neurosci. Res. 63: 10-16.

Gałecki P, Orzechowska A, Berent D, Talarowska M, et al. (2013). Vascular endothelial growth factor receptor 2 gene (KDR) polymorphisms and expression levels in depressive disorder. J. Affect. Disord. 147: 144-149.

Holmes DI and Zachary IC (2008). Vascular endothelial growth factor regulates stanniocalcin-1 expression via neuropilin-1. dependent regulation of KDR and synergism with fibroblast growth factor-2. Cell Signal. 20: 569-579.

lannolo G, Sciuto MR, La Rosa C and Conticello C (2011). MARCH-I expression in cord blood CD34+KDR+ cells. Clin. Biochem. 44: 725-727.

Kim CW, Son KN, Choi SY and Kim J (2006). Human lactoferrinupregulates expression of KDR/Flk-1 and stimulates VEGF-Amediated endothelial cell proliferation and migration. FEBS Lett. 580: 4332-4336.

Lortet-Tieulent J, Soerjomataram I, Ferlay J, Rutherford M, et al. (2014). International trends in lung cancer incidence by histological subtype: adenocarcinoma stabilizing in men but still increasing in women. Lung Cancer 84: 13-22.

Renouf DJ, Tang PA, Hedley D, Chen E, et al. (2014). A phase II study of erlotinib in gemcitabine refractory advanced pancreatic cancer. Eur. J. Cancer 50: 1909-1915.

Rinkevich FD, Su C, Lazo TA, Hawthorne DJ, et al. (2012). Multiple evolutionary origins of knockdown resistance (kdr) in pyrethroid-resistant Colorado potato beetle, Leptinotarsadecemlineata. Pest. Bioch. Physiol. 104: 192-200.

Santos SC, Miguel C, Domingues I, Calado A, et al. (2007). VEGF and VEGFR-2 (KDR) internalization is required for endothelial recovery during wound healing. Exp. Cell Res. 313: 1561-1574.

Tsuta K, Kawago M, Yoshida A, Sekine S, et al. (2014). Primary lung adenocarcinoma with morule-like components: a unique histologic hallmark of aggressive behavior and EGFR mutation. Lung Cancer 85: 12-18.

Vickers MM, Powell ED, Asmis TR, Jonker DJ, et al. (2012). Comorbidity, age and overall survival in patients with advanced pancreatic cancer - results from NCIC CTG PA.3: a phase III trial of gemcitabine plus erlotinib or placebo. Eur. J. Cancer 48: 1434-1442. 